\title{
Maintaining anorectal function in patients with rectal cancer using biofeedback training
}

\author{
Andrea M. Stringer ${ }^{1,2}$ \\ ${ }^{1}$ School of Pharmacy and Medical Sciences, University of South Australia, Adelaide, Australia; ${ }^{2}$ Adelaide Medical School, The University of Adelaide, \\ Adelaide, Australia \\ Correspondence to: Dr. Andrea M. Stringer. Senior Research Fellow, Musculoskeletal Biology Research Group, School of Pharmacy and Medical \\ Sciences, University of South Australia, North Terrace, Adelaide, SA 5000, Australia. Email: andrea.stringer@unisa.edu.au. \\ Provenance: This is an invited article commissioned by the Editorial Office of Annals of Translational Medicine. \\ Comment on: Liu L, Wu X, Liu Q, et al. The effect of biofeedback training on intestinal function among patients with middle and low rectal cancer: a \\ randomized controlled study. Ann Transl Med 2019;7:605.
}

Submitted Dec 02, 2019. Accepted for publication Dec 13, 2019.

doi: $10.21037 /$ atm.2019.12.84

View this article at: http://dx.doi.org/10.21037/atm.2019.12.84

Liu et al. (1) have studied the effects of biofeedback training in maintaining intestinal function in patients with rectal cancer. This study is important, as loss of intestinal control and function following sphincter preserving surgery can be common, and there is a growing number of articles investigating the effectiveness of biofeedback training with promising results $(1,2)$.

Over time, treatment of rectal cancer has improved, with sphincter preservation surgery becoming a feasible surgical option to restore bowel continuity, circumventing a permanent stoma. However, these surgical procedures can inadvertently adjust neural reflexes and control mechanisms of the lower bowel, resulting in changed bowel habits following surgery, known as anterior resection syndrome (ARS), which can occur in up to $90 \%$ of patients, and incorporates faecal incontinence (FI), increased bowel frequency and emptying difficulties. Options for treatment are limited, to the point that ARS and FI seem to be widely accepted as 'just the way it is'. Patient quality of life can be severely affected by bowel functional outcomes, with multiple studies evaluated, demonstrating a direct relationship (3).

The study by Liu and colleagues is a prospective randomised controlled study, whereby 126 patients with middle or low rectal cancer were randomly assigned to blank control, pelvic floor muscle exercise control, and biofeedback training intervention groups, consisting of routine nursing, a nurse- and video-guided series of pelvic floor muscle tightening exercises, and placement of electrodes forming a circuit on body surface and in lower rectum, respectively (1). Biofeedback training occurs when the participant modifies the way they contract their sphincter muscles based on readouts from an electromyograph (EMG) from the electrode circuit, whereby improving the level of control patients can gain over bowel continence and emptying, potentially improving the quality of life significantly.

The authors demonstrate that whilst biofeedback training cannot completely ameliorate intestinal dysfunction in patients with middle and low rectal cancer, it does reduce the severity and dysfunction and promotes intestinal functional recovery during the course of treatment (1), which can be considered a step forward in the treatment of ARS and FI, especially when compared to current treatments, which may or may not be effective, depending on the individual.

The authors repeated the biofeedback training over multiple sessions, with $3 \times 20$ minute sessions per week over 4 consecutive weeks considered one course of treatment, with 4 courses of treatment conducted in total, starting the first course in week 1 of the study, the second course 1 month after radical surgery, the third course was from stoma returning surgery, and the fourth course was 3 months following stoma returning surgery. Intestinal function was also assessed over multiple sessions, beginning prior to intervention, followed by 1 day prior to radical 
surgery, then 4 days, 3 months and 9 months following stoma returning surgery using patient questionnaires evaluating defecation frequency and urgency, effects of diet of defecation and abnormal feeling during defecation. Evaluation of these questionnaires (despite the inherent limitations and variance of interpretation of questions within questionnaires) did, however, show a significant difference in scores between the biofeedback group and blank control group $(\mathrm{P}<0.05)$. The biofeedback group and the pelvic floor muscle exercise group when comparing defecation frequency and urgency, and abnormal feeling during defecation were also significantly different $(\mathrm{P}<0.05)(1)$, which is a potentially promising step forward in improving intestinal function following surgery for rectal cancer. Restoring surgery in rectal cancer patients can improve the sensory function of the rectum (4).

Despite the promising nature of the findings from this biofeedback training trial, there are a number of limitations to this study. Questionnaires where patients are asked to answer a series of questions can result in a degree of variance, depending on the patient's interpretation of terms such as 'normal'. Other issues highlighted by the authors include the pelvic floor muscle exercises being conducted at home by patients following in-clinic instruction, and determining whether or not full patient compliance was achieved with this method. However, any promising result should be taken in a positive light, with plans for future studies to overcome such limitations, and expand studies to include multiple centres with larger patient numbers.

In conclusion, biofeedback training may become a viable option for treating intestinal dysfunction following rectal cancer surgery, and has shown some promise in improving measures of intestinal dysfunction in a small group of

Cite this article as: Stringer AM. Maintaining anorectal function in patients with rectal cancer using biofeedback training. Ann Transl Med 2020;8(4):63. doi: 10.21037/ atm.2019.12.84 patients with middle and low rectal cancer.

\section{Acknowledgments}

None.

\section{Footnote}

Conflicts of Interest: The author has no conflicts of interest to declare.

Ethical Statement: The author is accountable for all aspects of the work in ensuring that questions related to the accuracy or integrity of any part of the work are appropriately investigated and resolved.

\section{References}

1. Liu L, Wu X, Liu Q, et al. The effect of biofeedback training on intestinal function among patients with middle and low rectal cancer: a randomized controlled study. Ann Transl Med 2019;7:605.

2. Liang Z, Ding W, Chen W, et al. Therapeutic Evaluation of Biofeedback Therapy in the Treatment of Anterior Resection Syndrome After Sphincter-Saving Surgery for Rectal Cancer. Clin Colorectal Cancer 2016;15:e101-7.

3. Keane C, Sharma P, Yuan L, et al. Impact of temporary ileostomy on long-term quality of life and bowel function: a systematic review and meta-analysis. ANZ J Surg 2019. [Epub ahead of print].

4. Remes-Troche JM, De-Ocampo S, Valestin J, et al. Rectoanal reflexes and sensorimotor response in rectal hyposensitivity. Dis Colon Rectum 2010;53:1047-54. 